\title{
Serum-Reduced and Serum-Free Media for Differentiation of Caco-2 Cells
}

\author{
Simonetta Ferruzza, Carlotta Rossi, Yula Sambuy, and Maria Laura Scarino \\ National Research Institute on Food and Nutrition (INRAN), Rome, Italy
}

\begin{abstract}
Summary
Human intestinal Caco-2 cells were differentiated using serum-reduced medium with fetal bovine serum (FBS) added only to the basolateral (BL) medium, and four serum-free media, containing insulin, transferrin, selenium (ITS), or MITO+ ${ }^{T M}$ serum extender (ITS plus growth factors), with or without addition of a lipid mixture, respectively. Differentiation was assessed by monitoring monolayer permeability, alkaline phosphatase and sucrase activities, and the transport of digoxin and cephalexin. Notably, the serum-reduced protocol produced results that were comparable to cells differentiated in the control medium and should be recommended as an alternative to the use of $10 \% \mathrm{FBS}$ in both apical (AP) and BL media. ITS serum-free medium elicited permeability values and cephalexin transport similar to control cells. MITO+ ${ }^{T M}$ medium was the most efficient in promoting the two transport activities investigated, and it should be further evaluated with a larger set of substances, although its undisclosed composition represents a limit that may override these advantages.
\end{abstract}

Keywords: alternative methods, intestinal permeability, brush border enzymes, MDR-1/P-glycoprotein, peptide transporter-1, serum-free medium

\section{Introduction'}

To maintain, propagate, and differentiate human and animal cells in vitro, the culture medium must be supplemented with serum, either of human or animal origin. Serum is a very complex mixture containing a large number of components, including growth factors, transport proteins, trace elements, hormones, etc., essential for cell proliferation and differentiation. The most commonly used serum in cell culture media is fetal bovine serum (FBS) because of its low $\gamma$-globulin content and its strong growth-promoting activity (Gstraunthaler et al., 1999). However, the use of FBS as a cell culture media supplement raises several concerns, based on ethical, scientific, safety, and commercial considerations:

1) Collection of serum causes suffering to the unborn calf, unless strictly controlled procedures are used (van der Valk et al., 2004).

2) Composition of serum is still partially undefined and, more importantly, it can vary greatly from supplier to supplier and, within the same brand, from batch to batch (Rolleston, 1999). Such variability can affect cell culture conditions, ultimately causing uncontrolled variations in experimental results (Sambuy et al., 2005; Zucco et al., 2005; Brindley et al., 2012).
3) Despite routine testing procedures, FBS can still carry unidentified pathogens. To avoid contaminations, it should therefore be replaced by animal-free culture supplements in the manufacture of biological medicinal products (vaccines, monoclonal antibodies, hormones) and in the media of human cell cultures for tissue engineering, cell-based therapy, etc. Moreover, guidelines for good cell culture practice recommend, whenever possible, the use of serum free-media (Coecke et al., 2005). In 2008 the European Centre for Alternative Methods Scientific Advisory Committee issued a statement about the use of FBS and other animal-derived supplements, in favor of the use of specified synthetic supplements to satisfy both reproducibility and animal welfare ethics (http://ecvam.jrc.it/publication/ESAC28_statement_ FCS_20080508.pdf).

4) The fast growing cell therapy industry (CTI) is already facing the problem of limited availability of FBS; serum production and current serum stocks soon will be unable to support the CTI, and a significant raise in the price of good manufacturing practice-grade serum has already occurred in recent years (Brindley et al., 2012).

The human intestinal Caco-2 cell line (Pinto et al., 1983) has been used extensively during the past 30 years as the best avail-

\footnotetext{
Received November 5, 2012; accepted in revised form December 19, 2012

1 Abbreviations

ALP, alkaline phosphatase; AP, apical; BL, basolateral; BSA, bovine serum albumin; CTI, cell therapy industry;

DMEM, Dulbecco's Modified Eagle's Medium; EGF, epidermal growth factor; FBS, fetal bovine serum;

ITS, insulin-transferrin-selenium; MDR1, multidrug resistance-1; P-gp, P-glycoprotein; SUC, sucrase;

TEER, trans-epithelial electrical resistance; T3, triiodothyronine
} 
able in vitro model of the absorptive enterocyte. Caco- 2 cells undergo in culture a process of spontaneous differentiation leading, after three weeks, to a phenotype expressing many morphological and functional characteristics of mature small intestinal enterocytes (Hidalgo et al., 1989; Delie and Rubas, 1997; Sun et al., 2002). However, these characteristics can vary from laboratory to laboratory, due to both the origin of the cell lines and the different protocols used to maintain these cells in culture (Sambuy et al., 2005). Among the sources of variability in the performance and in the degree of differentiation of Caco-2 cells is the medium composition, which is deeply affected by its more undefined supplement, namely FBS. Therefore, in addition to ethical reasons for the reduction of FBS use, there are strong scientific motivations for the development of reliable serum-free, chemically-defined media for differentiation of Caco-2 cells.

It has been more than twenty years since the first reports of a serum-free medium for Caco-2 cells (Jumarie and Malo, 1991), and the use of this cell line has grown exponentially over this period (PubMed records: less than 200 up to the end of 1991 and almost 10,500 to December 2012). Despite that, a very small number of laboratories have adopted a serum-free protocol for differentiation of this popular cell line. The introduction of a commercially available Caco- 2 assay system, the BD Biocoat ${ }^{\mathrm{TM}}$ HTS, employing filters coated with fibrillary collagen I and using a differentiation medium containing a mixture of hormones and growth factors (MITO+ ${ }^{\mathrm{TM}}$ serum extender), reported to allow full differentiation of Caco- 2 cells in 3 days, compared to the 21-day standard protocol, has encouraged research to verify the company's claims. Overall, the results of these studies concluded that the 3-day accelerated differentiation protocol did not allow full expression of active membrane transporters and differentiation-dependent enzyme activities, although passive permeability properties were comparable to those obtained with the standard 21-day protocol employing medium supplemented with 10\% FBS (Chong et al., 1997; Miret et al., 2004; Yamashita et al., 2002). It is unfortunate, however, that these studies only compared the 3-day serum-free protocol with the standard 21-day $10 \%$ FBS-medium, while failing to investigate the serum-free protocol over an extended period of time.

The aim of the present study was to investigate the effects of distinct serum-free or serum-reduced media on the differentiation of Caco- 2 cells. To improve reproducibility of the cell model, a new cell-maintenance protocol (Natoli et al., 2011, 2012) that improves the homogeneity of Caco- 2 cells, was employed to propagate Caco- 2 cells. In addition, to reproduce the asymmetry existing in vivo, reflected by the different composition of fluids that come in contact with the apical (AP) and basolateral (BL) membranes of intestinal cells, 10\% FBS or other serum replacements were added only to the BL compartment, and their effects were compared to the standard, symmetric protocol $(10 \%$ FBS added to both compartments). In serum-free media, serum was replaced by the insulin-transferrin-selenium (ITS) mixture, originally used for Caco-2 differentiation (Jumarie and Malo, 1991), or with a commercial preparation of ITS enriched with growth factors and hormones, the MITO ${ }^{\mathrm{TM}}$ serum extender $\left(\mathrm{MITO}+{ }^{\mathrm{TM}}\right)$. To provide cells with lipids, a BSA conjugated- lipids mixture was prepared according to Morel et al. (2004) and added to both ITS and MITO+ ${ }^{\mathrm{TM}}$ serum-free media.

The degree of differentiation achieved by culturing Caco- 2 cells in serum free- and serum-reduced media was monitored by a set of parameters related to intestinal function. The establishment of the intestinal barrier was measured by transepithelial electrical resistance (TEER) and by the paracellular passage of mannitol (Madara, 1998). Maturation of brush border enzymes was assessed by the activity of sucrase (SUC) and alkaline phosphatase (ALP), two widely used markers of intestinal differentiation (Van Beers et al., 1995; Hodin et al., 1995; Matsumoto et al., 1990). Finally, the transport function of the absorptive enterocytes was monitored by measuring the activity of the AP oligopeptide transporter 1, PEPT1 (Walter and Kissel, 1995), also involved in the transport of important drug families, including antibiotics, antivirals, and anticancer agents (Nielsen and Brodin, 2003) and of the AP pump Multi Drug Resistance-1 (MDR1) / P-glycoprotein (P-gp) active in the AP efflux of orally ingested xenobiotics and drugs (Hunter et al., 1993). The purpose of this work was to provide recommendations for the use of serum-free or serum-reduced protocols for differentiation of Caco- 2 cell monolayers, with the ultimate aim of contributing to the reduction in the use of serum as culture medium supplement. This aim is in line with the $3 R$ principles, both in terms of refinement of methods employing animal-derived products and of reduction of the suffering of animals slaughtered to obtain FBS.

\section{Materials and methods}

\section{Cell line maintenance and differentiation}

The human intestinal Caco-2 cell line was originally obtained from Alain Zweibaum (Institut National de la Santé et de la Recherche Médicale, Villejuif, France). Cells were maintained at $37^{\circ} \mathrm{C}$ in $90 \%$ air- $10 \% \mathrm{CO}_{2}$ atmosphere in Dulbecco's Modified Eagle's Medium (DMEM) containing $25 \mathrm{mM}$ glucose, $3.7 \mathrm{~g} / \mathrm{l} \mathrm{NaHCO} 3,4 \mathrm{mM}$ L-glutamine, $1 \%$ non-essential amino acids, $100 \mathrm{U} / 1$ penicillin and $100 \mu \mathrm{g} / 1$ streptomycin (from now on referred to as complete DMEM), supplemented with $10 \%$ heat-inactivated FBS (FBS-Hyclone Laboratories, Logan, UT, USA). Cells were sub-cultured according to the low-density (LD) protocol described in (Natoli et. al., 2012). Briefly, cells were seeded at $6.2 \times 10^{3}$ cells $/ \mathrm{cm}^{2}$ and sub-cultured for at least 10 passages at 50\% confluence (approximately $5.4 \times 10^{4}$ cells/ $\mathrm{cm}^{2}$ ). Subsequently, cells were expanded to obtain a large stock of cells that was stored in liquid nitrogen. All experiments were performed within a range of 4 passages after cell thawing. All reagents were from Sigma-Aldrich (Milan, Italy), unless otherwise stated.

For differentiation cells were seeded on polycarbonate filters, $12 \mathrm{~mm}$ diameter, $0.4 \mu \mathrm{M}$ pore diameter (Transwell ${ }^{\circledR}$, Corning Inc., Lowell, MA, USA) at a density of $3.5 \times 10^{5}$ cells $/ \mathrm{cm}^{2}$ in complete DMEM containing $10 \%$ FBS in both AP and BL compartments (day 1); cells reached confluence after 2 to 3 days, and on day 3 they were either kept in the same medium (FBS 
Symmetric control) or transferred to complete DMEM with the addition in the BL compartment of either 10\% FBS (FBS Asymmetric control) or different serum substitutes, with or without addition of a lipid mixture, and allowed to differentiate until day 21 from seeding. Medium was regularly changed three times a week. Serum substitutes were: ITS (final concentration $10 \mathrm{mg} / \mathrm{l}$ insulin, $5.5 \mathrm{mg} / \mathrm{l}$ transferrin, $6.7 \mu \mathrm{g} / \mathrm{l}$ sodium selenite; GIBCO-Invitrogen, San Giuliano Milanese, Italy) or the commercial mixture MITO+ ${ }^{\mathrm{TM}}$ serum extender (Becton Dickinson, Bedford, MA, USA), containing ITS, epidermal growth factor (EGF), Endothelial Cell Growth Supplement, triiodothyronine (T3), hydrocortisone, progesterone, estradiol-17 $\beta$, and $\mathrm{O}$-phosphoryl-ethanolamine. ITS and $\mathrm{MITO}+{ }^{\mathrm{TM}}$ media were used with or without addition of a lipid mixture containing $0.2 \mathrm{mM}$ palmitic acid, $0.4 \mathrm{mM}$ oleic acid, and $0.04 \mathrm{mM}$ cholesterol in $1 \%(\mathrm{w} / \mathrm{v})$ fatty acid-free bovine serum albumin (BSA) as carrier (4:1 ratio of fatty acids to albumin), prepared as previously reported (Morel et al., 2004).

\section{Experimental media}

- FBS Symmetric: complete DMEM supplemented with $10 \%$ FBS in both AP and BL compartments;

- FBS Asymmetric: complete DMEM in AP compartment, supplemented with $10 \%$ FBS in BL compartment only;

- ITS: complete DMEM in AP compartment, supplemented with ITS serum replacement in BL compartment only;

- ITS Lipids: complete DMEM in AP compartment supplemented with ITS and lipid mixture in BL compartment only;

- MITO+: complete DMEM in AP compartment, supplemented with MITO+ ${ }^{\mathrm{TM}}$ serum extender in BL compartment only;

- MITO+ Lipids: complete DMEM in AP compartment, supplemented with $\mathrm{MITO}+{ }^{\mathrm{TM}}$ serum extender and lipid mixture in BL compartment only.

\section{Permeability experiments}

Permeability of the cell monolayer was monitored at 15 and 21 days from seeding by measuring TEER and the AP to BL passage of D-[1- $\left.{ }^{14} \mathrm{C}\right]$-mannitol (specific activity $1.887 \mathrm{GBq} /$ mmol-Perkin Elmer, Milano, Italy). TEER was measured at $37^{\circ} \mathrm{C}$ using a commercial apparatus (Millicell ERS; Millipore, Bedford, MA, USA), as previously described (Ferruzza et al., 2003). D-[1- $\left.{ }^{14} \mathrm{C}\right]$-mannitol was added to $10 \mu \mathrm{M}$ D-mannitol in DMEM (final specific activity $1.52 \mathrm{MBq} / \mathrm{mmol}$ ) and loaded into the AP compartment. After 1 hour of incubation, radioactivity was measured in the BL compartment using a liquid scintillation counter (1450 LSC Microbeta Trilux Wallac Luminescent Counter; Perkin Elmer, Monza, Italy). Mannitol passage was expressed as apparent permeability $\left(\mathrm{P}_{\text {app }}\right)$ measured in $\mathrm{cm} \mathrm{x} \mathrm{s}^{-1}$ (Ranaldi et al., 2003).

\section{Enzyme activities}

ALP and SUC activities were measured after 15 and 21 days from seeding using in situ assays performed on live cells as previously reported (Ferruzza et al., 2012a). Briefly, ALP activity was measured on live cells using p-nitrophenyl phosphate as substrate and measuring the activity at $\mathrm{pH} 9.3$ by the release of p-nitrophenol, detected at $405 \mathrm{~nm}$ in a microplate multifunctional reader (TECAN, Infinite 200, Austria) and converted to concentration after blank subtraction with reference to a standard curve. For the SUC assay, release of glucose from sucrose hydrolysis in the AP compartment of live cells was measured employing the fluorescent Amplex Red Glucose/Glucose Oxidase Kit (Molecular Probes, Eugene OR). In the assay, glucose oxidase reacts with D-glucose to form D-gluconolactone and $\mathrm{H}_{2} \mathrm{O}_{2}$. In the presence of horseradish peroxidase, $\mathrm{H}_{2} \mathrm{O}_{2}$ reacts with the Amplex Red reagent in 1:1 stoichiometry to generate the red-fluorescent oxidation product, resorufin, which was detected in the TECAN microplate reader adjusted for excitation at $530 \mathrm{~nm}$ and emission at $590 \mathrm{~nm}$, and converted to concentration after blank subtraction with reference to a standard curve. In both assays, enzyme activities were calculated from the angular coefficient of the linear slope of product appearance and expressed as $\mathrm{mU}=\mathrm{nmol}$ of reaction product $/ \mathrm{min}$ at $37^{\circ} \mathrm{C}$, and normalized to $\mathrm{mg}$ of cellular protein, measured according to Lowry et al. (1951).

\section{Transport experiments}

Transport experiments of cephalexin and digoxin were performed at day 21 in cells differentiated in different experimental media. The integrity of the tight junctions of cells was monitored before and at the end of transport experiments by measuring TEER.

Cephalexin transport was assayed in $\mathrm{AP}$ to $\mathrm{BL}$ direction in Hank's balanced salt solution (HBSS: $137 \mathrm{mmol} / \mathrm{l} \mathrm{NaCl}$, $5.36 \mathrm{mmol} / \mathrm{l} \mathrm{KCl}, 0.44 \mathrm{mmol} / 1 \quad \mathrm{KH}_{2} \mathrm{PO}_{4}, 0.34 \mathrm{mmol} / \mathrm{l}$, $\mathrm{Na}_{2} \mathrm{HPO}_{4} 1 \mathrm{mmol} / \mathrm{l} \mathrm{CaCl}, 1 \mathrm{mmol} / 1 \mathrm{MgCl}_{2}, 5.6 \mathrm{mmol} / \mathrm{l} \mathrm{glu}-$ cose), buffered with either $10 \mathrm{mmol} / 1$ morpholino-ethanesulfonic acid monohydrate ( $\mathrm{pH}$ 6.5) in the AP compartment or with $10 \mathrm{mmol} / \mathrm{l}$ N-2-hydroxyethyl-piperazine-N-4-butanesulfonic acid ( $\mathrm{pH} 7.4$ ) in the BL compartment. A pre-warmed solution of cephalexin hydrate $(100 \mu \mathrm{M}$ in HBSS $)$ was added to the AP compartment and transport was assayed by collecting and totally replacing the $\mathrm{BL}$ medium at set time intervals up to $60 \mathrm{~min}$. Cephalexin was measured in the BL medium by LC/ MS and transport was expressed as $\mathrm{P}_{\text {app }}$. Briefly, cephalexin was analyzed with an UltraPerformance LC system (Waters Acquity UPLC, Milford, MA) connected to a triple quadrupole MS system (API 4000, AB Sciex, The Netherlands) equipped with an ionspray probe, operating in the positive ion mode (ion source temperature set at $550^{\circ} \mathrm{C}$ ). D3-testosterone was used as internal standard. The MS system was operated in the multiple-reaction monitoring mode, with the transition 348.2/158.1. A $10 \mu \mathrm{l} \mathrm{sam-}$ ple was loaded on a VanGuard BEH C18 precolumn $(1.7 \mu \mathrm{m}$, Waters) connected to an Acquity UPLC BEH C18 column, (30 x $2.1 \mathrm{~mm}$ I.D, $1.7 \mu \mathrm{m}$, Waters) maintained at $50^{\circ} \mathrm{C}$. Mobile phase A consisted of $0.1 \%$ formic acid and 5\% acetonitrile in MilliQ, and mobile phase B consisted of acetonitrile. A 1.1-min gradient was used in which the initial proportion mobile phase $\mathrm{B}$ was $5 \%$ and linearly increased to $95 \% \mathrm{~B}$ in $0.65 \mathrm{~min}$ and held for 0.3 min, then returning to $5 \% \mathrm{~B}$ in $0.15 \mathrm{~min}$ at a flow rate of $0.85 \mathrm{ml}$ $\mathrm{x} \mathrm{min}{ }^{-1}$. The limit of quantification for cephalexin was $5 \mathrm{nM}$. 
Digoxin transport experiments were performed in AP to BL and BL to AP direction in HBSS at $\mathrm{pH} 7.4$ in both compartments (no $\mathrm{pH}$ gradient), with or without verapamil as specific inhibitor of P-gp. $\left[{ }^{3} \mathrm{H}(\mathrm{G})\right]$ Digoxin (specific activity $1.48 \mathrm{TBq} /$ mmol-PerkinElmer, Milano, Italy) was dissolved in HBSS $\mathrm{pH} 7.4$ containing $10 \mu \mathrm{M}$ digoxin to a final specific activity of $3.7 \mathrm{MB} / \mathrm{mmol}$. Cells were pre-incubated for $30 \mathrm{~min}$ in $\mathrm{HB}$ $\mathrm{SS}$ with or without addition of $200 \mu \mathrm{M}$ verapamil $\mathrm{HCl}$ in the AP and BL compartment. The transport of $10 \mu \mathrm{M}\left[{ }^{3} \mathrm{H}(\mathrm{G})\right] \mathrm{Dig}-$ oxin \pm verapamil hydrochloride was assayed by collecting and replacing the donor compartment medium at set times up to $60 \mathrm{~min}$. Radioactivity was detected by a scintillation counter (1450 LSC Microbeta Trilux Wallac Luminescent Counter; Perkin Elmer, Monza, Italy). All transport experiments were performed under "sink" conditions. Apparent permeability ( $\mathrm{Papp}$ ) was calculated from the linear appearance curve of the transported molecule in the acceptor compartment (Ranaldi et al., 2003).

\section{Statistical analysis}

Statistical analysis was performed by one-way or two-way ANOVA, followed by Bonferroni's post-hoc test (GraphPad Prism 5 Software).

\section{Results}

\subsection{Permeability of Caco-2 cell monolayers}

Permeability changes during Caco- 2 differentiation were monitored at days 15 and 21 by two parameters, TEER, an electri- cal measure of ion fluxes across the monolayers, and AP to BL passage of ${ }^{14} \mathrm{C}$-mannitol, a molecule that crosses the monolayer only by the paracellular route (Fig. 1A,B). As shown in Figure $1 \mathrm{~A}$, at day 15 TEER values of Caco-2 cells differentiated both in FBS Asymmetric and ITS media, were slightly but significantly higher, while both MITO+ ${ }^{\mathrm{TM}}$ and $\mathrm{MITO}+{ }^{\mathrm{TM}}$ Lipids media resulted in significantly lower values than obtained in FBS Symmetric control medium. At day 21, cells differentiated in FBS Asymmetric medium still showed significantly higher TEER values, while cells differentiated in ITS Lipids, MITO+ ${ }^{\mathrm{TM}}$ and MITO ${ }^{\mathrm{TM}}$ Lipids showed significantly lower values compared to cells in FBS Symmetric control medium.

Mannitol permeablity (Fig. 1B) reached similar values at day 15 in cells differentiated in all experimental media, with the exception of media supplemented with lipids mixture (ITS Lipids and MITO $+{ }^{\mathrm{TM}}$ Lipids) that showed significantly higher values compared to FBS Symmetric control cells. Caco-2 differentiated in all other media consistently showed similar and nonsignificantly different mannitol permeability values $(p>0.01)$, compared to control cells in FBS Symmetric medium.

Taken together, data on permeability properties indicate that by day 15 , functional maturation of Caco-2 tight junctions was achieved under all tested experimental conditions.

\subsection{Brush border enzyme activities}

Enzyme activities that are well established markers of intestinal differentiation in vivo and have often been used to monitor Caco-2 differentiation in vitro (Van Beers et al., 1995; Matsumoto et al., 1990), were measured to follow cell differentiation in serum-free or serum-reduced medium. ALP and SUC

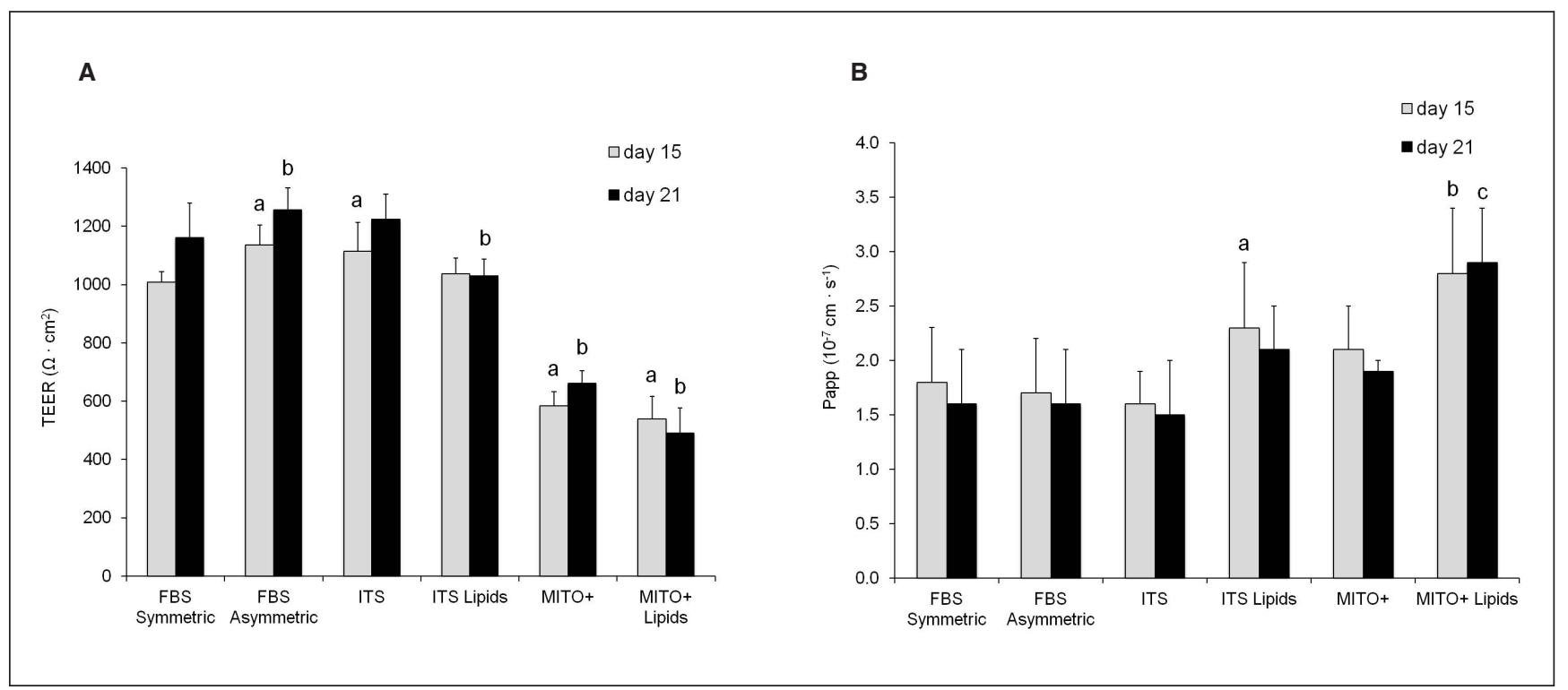

Fig. 1: Permeability of Caco-2 cell monolayers at 15 and 21 days of differentiation in serum-reduced and serum-free media TEER (A) and trans-epithelial mannitol passage (B) were measured in Caco-2 cells on days 15 and 21 of differentiation in culture media of different composition. Data are mean \pm SD of 5 independent experiments performed in triplicate. Statistical significance was determined by two-way ANOVA followed by Bonferroni's post-hoc test. A, $p<0.001$ (a) at day 15 versus FBS Symmetric; $p<0.001$ (b) at day 21 versus FBS Symmetric; B, $p<0.05$ (a), $p<0.001$ (b) at 15 day versus FBS Symmetric medium; $p<0.001$ (c) at day 21 versus FBS Symmetric medium. 
A

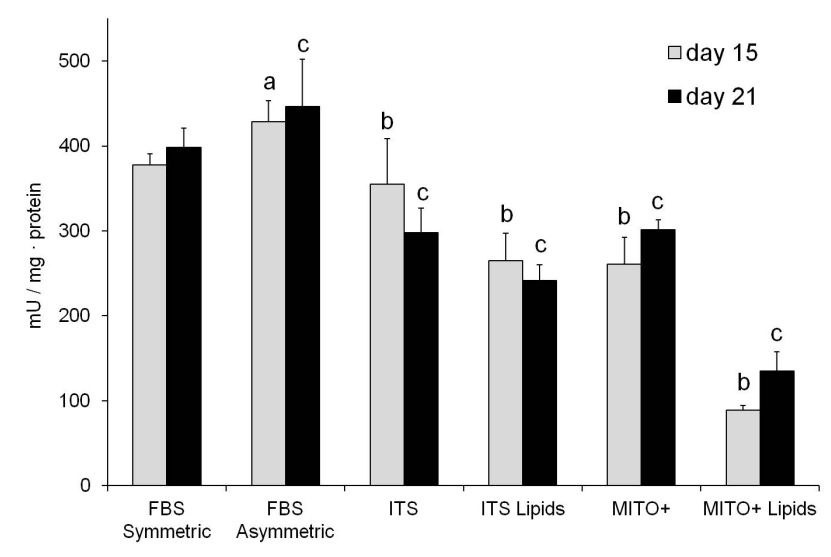

B

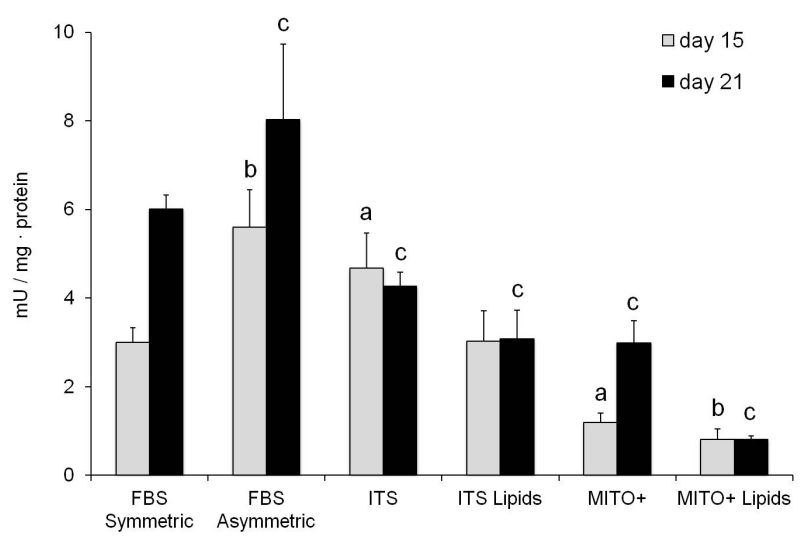

Fig. 2: Effect of medium composition on the enzymatic activity of brush border

Alkaline phosphatase (A) and sucrase (B) were measured in Caco-2 cells on days 15 and 21 of differentiation in culture media of different composition. Data are means $\pm S D$ of 4 independent experiments in triplicate. Statistical significance was determined by two-way ANOVA followed by Bonferroni's post-hoc test. $A, p<0.01$ (a), $p<0.001$ (b) at day 15 versus FBS Symmetric; $p<0.001$ (c) at day 21 versus FBS Symmetric medium. $B, p<0.01$ (a), $p<0.001$ (b) at day 15 day versus FBS Symmetric medium; $p<0.001$ (c) at day 21 versus FBS Symmetric medium.

activities were measured in situ on live Caco- 2 cells after 15 and 21 days in culture, and results are shown in Figures 2A and $\mathrm{B}$, respectively.

No major increase in ALP activity was observed between days 15 and 21 in Caco- 2 cells differentiated in all experimental media (Fig. 2A). On day 15 and day 21 cells differentiated in FBS Asymmetric medium showed significantly higher values, while cells differentiated in all other serum-free media showed significantly lower values compared to cells in FBS Symmetric control medium.

Cells differentiated in FBS Asymmetric medium showed higher values of SUC activity at both days 15 and 21, which was significantly different from activity of cells in FBS Symmetric medium. When serum-free media were used to differentiate Caco-2 cells, SUC activity showed significantly lower values compared to FBS Symmetric control cells at day 21 .

\subsection{Apical transporters activities}

The activity of two AP membrane transporters, PEPT1 and MDR1/P-gp as reference transporters for AP uptake and efflux, respectively, were measured in Caco- 2 at the end of differentiation (day 21), employing as specific substrates the $\beta$-lactam antibiotic cephalexin and digoxin, respectively. Since lipid addition to serum-free media, a time consuming and cumbersome procedure, was shown in previous experiments not to improve the differentiation of Caco- 2 cells, lipids mixture was omitted from serum-free media in further experiments.

Cephalexin transport in the AP to BL direction in Caco-2 differentiated for 21 days is shown in Figure 3. While cells differentiated in FBS (both Symmetric and Asymmetric) and ITS

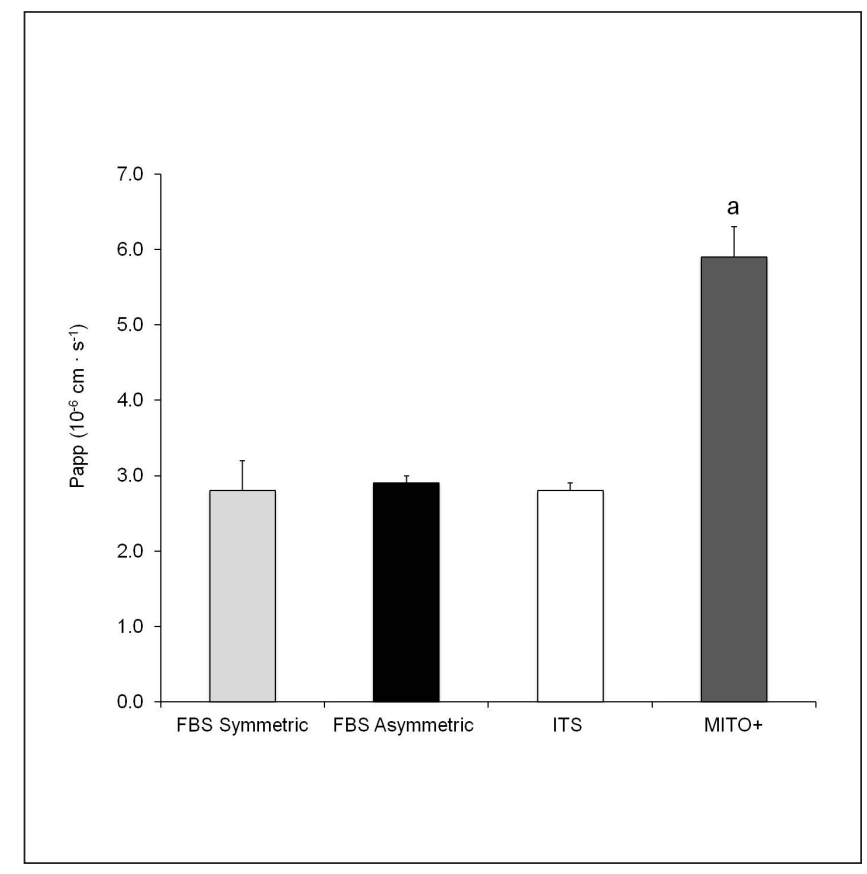

Fig. 3: Cephalexin transport in Caco-2 cells differentiated for 21 days in different experimental media PEPT1 activity was evaluated by measuring AP to BL transport of cephalexin in Caco-2 cells differentiated for 21 days in serum-reduced and serum-free media. Data are means $\pm S D$ of 3 experiments performed in duplicate. Statistical significance was determined by one-way ANOVA followed by Bonferroni's post-hoc test, $p<0.001(\mathrm{a})$. 
showed similar cephalexin transport activities, the use of MI$\mathrm{TO}+{ }^{\mathrm{TM}}$ medium significantly increased it with respect to FBS Symmetric control medium.

Activity of MDR1/P-gp, an AP directed efflux transporter located in the brush border membrane, was measured using ${ }^{3} \mathrm{H}$ digoxin as substrate and verapamil as specific inhibitor in secretory (BL to AP transport, Fig. 4A) and absorptive (AP to BL transport, Fig. 4B) directions. Verapamil inhibition was used to calculate the net AP efflux (secretory $\mathrm{P}_{\mathrm{app}}$ - verapamil inhibited secretory $\mathrm{P}_{\text {app }}$ ). As shown in Figure $4 \mathrm{~A}$, Caco-2 differentiated in FBS Asymmetric and MITO+ ${ }^{\mathrm{TM}}$ media showed significantly higher digoxin AP efflux compared to FBS Symmetric control medium, while ITS medium resulted in significantly lower AP efflux. When MDR1/P-gp activity was blocked by verapamil, residual AP efflux, due to passive diffusion and/or to different transporters, was similar in cells differentiated in FBS-containing media and in ITS medium, while it was slightly but significantly higher in both ITS and MITO+ media.

Absorption of digoxin in the AP to BL direction is shown in Figure 4B. Cells differentiated in media with FBS (both symmetric and asymmetric) showed similar absorption values. Conversely, cells in serum-free medium showed significantly higher absorption values. Inhibition of MDR1/P-gp AP counter-efflux activity by verapamil increased digoxin absorption to values that were non-significantly different under all conditions.

Differences in MDR1/P-gp activity can be highlighted by comparing net AP digoxin efflux, obtained by subtracting mean AP to BL transport from mean BL to AP transport. Net AP digoxin efflux ranked as follows: FBS Asymmetric: $14.48 \times 10^{-6} \mathrm{cmx} \mathrm{s}^{-1}$;MITO+: $10.63 \times 10^{-6} \mathrm{cmxs}^{-1}$; FBSSymmetric: $9.33 \times 10^{-6} \mathrm{~cm} \mathrm{x} \mathrm{s}^{-1}$; ITS: $5.33 \times 10^{-6} \mathrm{~cm} \mathrm{x} \mathrm{s}^{-1}$. This parameter indicates that FBS Asymmetric and MITO+ supplemented media performed better in promoting digoxin AP efflux, followed by FBS Symmetric and ITS medium, both showing lower net AP efflux values. Efflux ratio (mean secretory $\mathrm{P}_{\text {app }} /$ mean absorptive $\mathrm{P}_{\text {app}}$ ) was calculated for each experimental condition to evaluate specificity of MDR1/P-gp transport, and ranked the treatments as follows: FBS Asymmetric (14.75) = FBS Symmetric (14.11) $>>$ MITO $^{\mathrm{TM}}(6.67)>$ ITS (3.14). According to this parameter, the presence of FBS in the medium appears to promote specific digoxin efflux activity mediated by MDR1/P-gp.

\section{Discussion}

The first attempt to cultivate Caco- 2 cells in a serum-free, chemically defined medium dates back to the early 1990s (Jumarie and Malo, 1991). In this study, serum was replaced by ITS or by ITS supplemented with T3 and EGF, and differentiation of Caco-2 grown on plastic supports was monitored 21 days after seeding by structural and functional parameters. In cells differentiated in ITS medium, activity of ALP and SUC were lower as compared to control cells in symmetric $10 \%$ FBS medium. In those early days of Caco- 2 culture, these cells
A

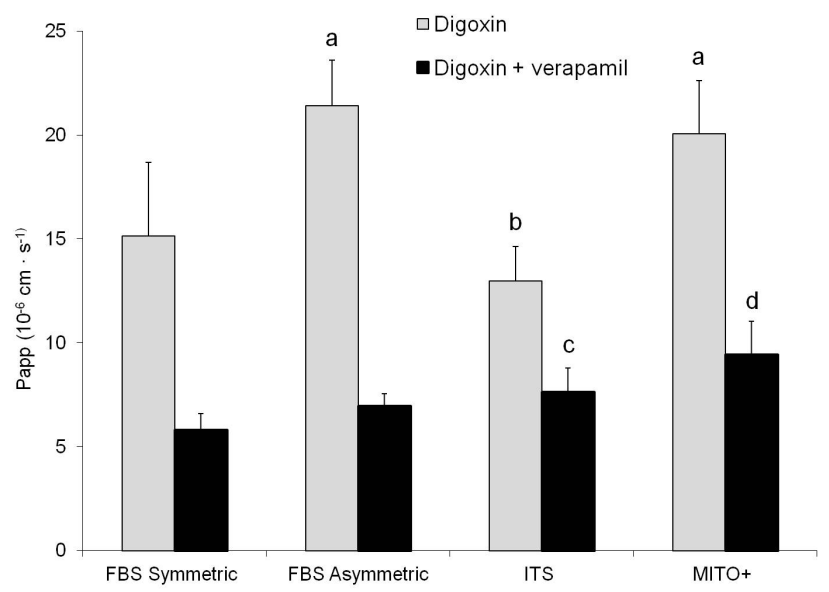

B

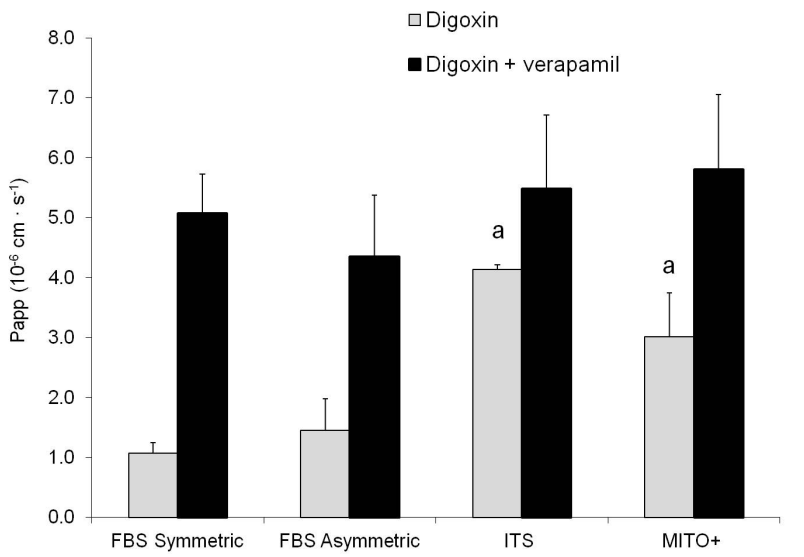

Fig. 4: Digoxin transport in Caco-2 cells differentiated for 21 days in different experimental media MDR1/P-gp activity was evaluated in Caco-2 cells differentiated for 21 days in serum-reduced and serum-free media, using ${ }^{3} \mathrm{H}$ digoxin as substrate with or without verapamil $(100 \mu \mathrm{M})$ as specific inhibitor in secretory (BL to AP transport, panel A) and absorptive (AP to BL transport, panel $B$ ) directions. Data are means $\pm S D$ of 3 independent experiments performed in triplicate. Statistical significance was determined by two-way ANOVA followed by Bonferroni's post-hoc test. A, Secretory transport: $p<0.001$ (a), $p<0.01$ (b) digoxin transport in all conditions versus FBS Symmetric medium; $p<0.05$ (c), $p<0.001$ (d) digoxin + verapamil transport in all conditions versus Symmetric medium. B, Absorptive transport: $p<0.001$ (a) digoxin transport in all conditions versus Symmetric medium; digoxin transport with verapamil showed non-significant differences in all conditions versus Symmetric medium. 
were grown and differentiated on impermeable supports (plastic or glass), such that permeability properties and establishment of functional tight junctions could not be investigated easily. With the advent of filter substrates, separating the AP from the BL compartment, thus allowing full differentiation and polarization of Caco-2 cell monolayers, permeability measurements, a critical feature of enterocyte differentiation, could be assessed by electrical (TEER) or functional (passage of molecules confined to the extracellular space) parameters (Hashimoto and Shimizu, 1993). A first attempt to differentiate Caco-2 cells on filters pre-coated with FBS and collagen and maintained in serum-free medium containing insulin and transferrin, however, did not allow establishment of cell polarity and monolayer integrity as measured by TEER and lucifer yellow permeability (Hashimoto and Shimizu, 1993). Monolayer permeability during differentiation was later measured in Caco-2 cells grown on filters in serum-free medium (FBS replaced by ITS in both compartments) (Baltes et al., 2004). In this work, all-trans retinoic acid added to serum-free medium (DMEM with $1 \%$ ITS) promoted the activity of ALP, a target of retinoid acid, but decreased paracellular permeability. However, this effect was ascribed to all-trans retinoic acid, since in control serum-free medium cells, TEER values increased and mannitol permeability decreased from day 1 to day 23 of differentiation, indicating that functional closure of tight junctions had regularly occurred. In addition, in the same study, ALP activity was about three times lower in ITS differentiated cells compared to control cells in FBS (Baltes et al., 2004). In our study, Caco- 2 cells differentiated on filters with $1 \%$ ITS supplemented only to the BL medium showed permeability properties very similar to both FBS supplemented media, i.e., symmetric and asymmetric FBS, but lower ALP and SUC activities. We had previously shown that permeability parameters (TEER and mannitol passage) in different Caco-2 substrains and clonal lines differentiated on polycarbonate filters using medium containing ITS and lipids mixture, reached, by 15 days after seeding, values similar to those of control cells in symmetric FBS medium (Ranaldi et al., 2003).

From these results we can conclude that addition of ITS to BL medium during Caco-2 differentiation is sufficient for the establishment of a functional tight monolayer, although expression of ALP and SUC activities are lower than in cells differentiated in FBS containing medium.

Differentiation in MITO+ ${ }^{\mathrm{TM}}$ medium resulted in cell monolayers showing mannitol permeability similar to control cells, but significantly lower TEER values. While mannitol permeability indicates paracelllular passage of molecules, TEER measures paracellular conductance of the monolayer and can be influenced by changes in the molecular composition of the junction (i.e., expression of different claudins), as well as by changes in the activity and/or selectivity of AP ionic channels (Madara, 1998; Van Itallie et al., 2008). The results thus indicate that the tight junctions were functionally close under all treatments but that MITO+ ${ }^{\mathrm{TM}}$ medium had produced changes in the molecular organization of the tight junction and/or had activated ionic channels, resulting in lower TEER than observed with the other treatments. As an example of this appar- ent discrepancy between paracellular permeability and TEER, the Caco-2 clonal line Caco-2/TC7 has similar mannitol permeability compared to the parental cell line, but exhibits lower TEER values (Zucco et al., 2005). However, lower TEER values may not represent a disadvantage, since the human small intestinal mucosa exhibits lower TEER values than Caco-2 cells differentiated in FBS containing medium (Le Ferrec et al., 2001). Thus, the lower TEER of Caco-2 cells differentiated in MITO+ may better represent the in vivo permeability than Caco- 2 cultured in serum containing (both symmetric and asymmetric) or ITS media.

Addition of T3 to ITS during the differentiation period has been reported to increase the activity of ALP in a dose-dependent way (Jumarie and Malo, 1994) and to stimulate SUC activity as well as sucrase-isomaltase mRNA and protein expression (Jumarie et al., 1996). The same authors, however, had previously reported that when a mixture of hydrocortisone (HC), EGF, and T3 was added to ITS, SUC activity actually decreased compared to ITS supplemented only with T3 (Jumarie and Malo, 1991). In the present work, the use of MITO+ ${ }^{\mathrm{TM}}$, which contains HC, EGF, and T3, did not increase SUC and ALP activities in differentiated Caco-2 cells to levels similar to those observed in control, FBS-containing medium. It is possible that, as previously reported (Jumarie and Malo, 1991), the presence of other components in MITO+ ${ }^{\mathrm{TM}}$ could contrast the effect of $\mathrm{T} 3$ when used alone. In addition, the concentration of the supplements in MITO+ is not disclosed by the supplying company, thus making it impossible to determine the dose-effects of single components and their synergies. In another report, EGF was shown to decrease SUC activity in the Caco-2/15 clonal cell line, acting on the processing of this enzyme at lower concentrations $(20 \mathrm{nM})$ and on its mRNA synthesis at higher concentrations (200 nM), while ALP activity was unaffected under the same conditions (Cross and Quaroni, 1991).

In the present study, the activity of the AP transporter PEPT1 was almost doubled as compared to symmetric FBS control medium when Caco- 2 cells were differentiated in $\mathrm{MITO}+{ }^{\mathrm{TM}}$ medium. Short-term (60 min) treatment of filter-grown Caco-2 cells with EGF and insulin from the BL side has previously been shown to increase the number of PEPT1 transporters in the AP membrane and to enhance PEPT1 activity (Nielsen et al., 2003). Conversely, long-term treatment with EGF ( $>5$ days) or T3 ( $>3$ days) has been shown to decrease PEPT1 activity, as reported in Nielsen and Brodin (2003). In the present work, Caco-2 cells were supplemented with MITO+ ${ }^{\mathrm{TM}}$ (which contains insulin, EGF, and T3) during the entire differentiation period (i.e., 21 days), and enhancement of the two active transporters was observed. Again, however, differences in concentration, time of exposure, and/or synergies with other components of MITO+ ${ }^{\mathrm{TM}}$ could explain differences from previous reports.

Increase in MDR1/P-gp activity by EGF has been demonstrated in rat primary hepatocytes maintained in serum-free culture (Hirsch-Ernst et al., 1995). P-gp activity and MDR1 expression also were increased significantly by progesterone in cultured porcine granulosa cells stimulated with luteinizing hormone (Fukuda et al., 2006). In our study, efflux activity of MDR1/P-gp in cells differentiated with MITO ${ }^{\mathrm{TM}}$ supplement- 
ed medium (containing both EGF and progesterone), was significantly higher than in ITS supplemented medium (containing only insulin as growth factor), suggesting also that in the Caco-2 cell model, EGF and progesterone could enhance MDR1/P-gp activity.

Lipid addition has been recommended in the preparation of optimized serum-free media (van der Valk et al., 2010). BSA-conjugated linoleic acid was employed as lipid supplement in a serum-free medium for Caco-2 differentiation, developed from a hybridoma serum-free medium (Halleux and Schneider, 1991). The BSA-cholesterol, palmitic and oleic acid mixture used as lipid supplement in this work has been used previously in studies aimed at clarifying the mechanisms of intestinal lipid absorption and metabolism in differentiated Caco-2 on filters (Beaslas et al., 2009) and was utilized by our group together with the ITS supplement to differentiate $\mathrm{Ca}$ co-2 cell lines of different origins (Ranaldi et al., 2003). In the present study, when we directly compared the effects of lipids on ITS and MITO+ ${ }^{\mathrm{TM}}$ supplemented media, lipid addition did not produce any significant improvement in the performance of the parameters under investigation. In addition, when added to MITO+ ${ }^{\mathrm{TM}}$ medium, the lipid mixture had a clear negative effect. A ready to use and chemically defined lipid mixture, produced by Sigma-Aldrich (L0288, Lipid Mixture 1) is now available on the market and could be a promising supplement for further investigations.

Taken together, the use of ITS as serum replacement resulted in permeability parameters and PEPT1 activity comparable to those obtained under standard culture conditions with $10 \%$ FBS added to both AP and BL compartments (FBS Symmetric), while ALP and SUC enzyme activities were lower. MDR1/Pgp activity in ITS differentiated cells was the lowest among all treatments. However, digoxin efflux ratio in cells differentiated with ITS medium, although the lowest (3.14) among all treatments, was indeed the closest to the values (ranging from 4.4 to 3.6) obtained in different segments of isolated intestinal human mucosa (Stephens et al., 2001). To try to improve the performance obtained with ITS, we tested the commercially available MITO+ ${ }^{\mathrm{TM}}$ serum extender, an enriched ITS preparation containing several other hormones and growth factors. MITO $+{ }^{\mathrm{TM}}$ serum extender is recommended by the producer for Balb/c-3T3 cell proliferation with the addition of $2.5 \%$ FBS. In addition, the Biocoat ${ }^{\mathrm{TM}}$ HTS system, which includes medium supplemented with MITO+ ${ }^{\mathrm{TM}}$ serum extender, is recommended for accelerated differentiation of Caco- 2 cells in 3 days (BD Biocoat ${ }^{\mathrm{TM}}$ HTS Caco-2 assay system). However, in this assay, differentiation medium also includes butyrate, which was shown to promote cellular differentiation but also causes toxicity when used longer than 5 days (Yamashita et al., 2002). Serum-free medium containing MITO+ ${ }^{\mathrm{TM}}$ serum extender has, to the best of our knowledge, never before been tested alone for differentiation of intestinal cell lines. Results obtained with the Caco- 2 cell line demonstrate that MITO+ ${ }^{\mathrm{TM}}$ medium enhanced PEPT1 activity compared to other experimental media and gave results similar to FBS Symmetric medium for MDR1/P-gp activity. Expression levels of PEPT1
mRNA by RT-PCR were found to be lower in Caco-2 cells than in the human jejunum (Hilgendorf et al., 2007) and in vivo permeability in the human jejenum was reported to be 1.56 . $10^{-4} \mathrm{~cm} \mathrm{x} \mathrm{s}^{-1}$ (Sun et al., 2002), 50-fold higher than in Caco-2 FBS symmetric medium and 24-fold higher than in Caco-2 $\mathrm{MITO}+{ }^{\mathrm{TM}}$ medium. Moreover, the digoxin efflux ratios in cells differentiated in MITO+ ${ }^{\mathrm{TM}}$ medium (6.67), similar to what was observed with ITS medium (3.14), were closer to those reported for the in vivo human intestinal mucosa (Stephens et al., 2001) than to cells differentiated in both FBS containing media (FBS Symmetric, 14.11; FBS Asymmetric, 14.75).

However, for the other parameters under investigation, $\mathrm{MITO}+{ }^{\mathrm{TM}}$ did not perform as well as ITS medium or as both FBS containing media. This indicates that the combination of different hormones and growth factors in MITO $+{ }^{\mathrm{TM}}$ serum extender, though enhancing the expression of some differentiation markers, may not be optimal for others. It should be noted, however, that the concentration of hormones and growth factors also may vary in different batches of FBS, according to the time of collection of the fetuses and/or the diet of the mother cows (van der Valk et al., 2004), and this may be one of the reasons for the heterogeneity of Caco- 2 cells cultured in different laboratories (Sambuy et al., 2005). On the other hand, the advantages of removing FBS, an animal-derived component of undefined composition, from cell culture medium appear to be far greater than the differences in the expression of single parameters in the overall performance of the cell line. Further studies using a wider range of molecules that permeate by distinct routes will be required to better evaluate the transport capacity of the Caco- 2 cell line differentiated in serum-free medium in order to reach conclusions on the strength of the different media composition for in vitro/in vivo predictions.

FBS was not replaced during propagation of Caco- 2 cells to avoid an additional experimental variable, since this phase has been shown to be very critical and can greatly influence further differentiation, as previously reported (Natoli et al., 2012). In addition, the amount of FBS used during the differentiation period (21 days with 3 medium changes a week) is far greater than required during the propagation of the cell line. However, it would be desirable, as a future goal, also to replace FBS during the growth phase and the initial attachment of Caco-2 cells destined to differentiation, to obtain a full serum-free protocol for the use of this cell line.

In addition to ethical and commercial considerations, the protocol tested in the present work is of important scientific relevance, since the use of media of chemically defined composition reduces the variability of experimental results and allows better control of the conditions of differentiation.

In conclusion, differentiation of Caco- 2 cells in ITS medium leads to the formation of a well-differentiated cell monolayer that can be used for most Caco-2 cell applications, while the uncertainty in the composition of the commercial MITO+ ${ }^{\mathrm{TM}}$ serum extender may represent a stronger point for not recommending its use than its capacity to enhance active transport activities.

Finally, we would like to stress that the use of FBS in asymmetric conditions for Caco- 2 differentiation gave better results 
than the standard FBS symmetric protocol. The asymmetric culture condition was originally used in previous studies (Morel et al., 2004; Beaslas et al., 2009) to mimic in vitro the physiological asymmetric intestinal environment existing in vivo, and a detailed protocol for its use has recently been published (Ferruzza et al., 2012b).

Although the first protocols to grow and differentiate Caco-2 in serum free media date back to the 1990's, this procedure is not yet established, and it is seldom used to differentiate this cell line in laboratories around the world. Thus, in addition to the serum-free protocols here tested, even the use of the FBS asymmetric protocol represents a substantial improvement to the traditional medium containing 10-20\% FBS in both compartments, by being more physiological, more economical, as it halves the consumption of serum, and more ethical, as it contributes to reducing the number of slaughtered bovine fetuses for serum production, thus complying with the 3 Rs principles.

\section{References}

Baltes, S., Nau, H., and Lampen, A. (2004). All-trans retinoic acid enhances differentiation and influences permeability of intestinal Caco-2 cells under serum-free conditions. Dev Growth Differ 46, 503-514.

Beaslas, O., Cueille, C., Delers, F., et al. (2009). Sensing of dietary lipids by enterocytes: a new role for SR-BI/CLA-1. PLoS One 4, e4278.

Brindley, D. A., Davie, N. L., Culme-Seymour, E. J., et al. (2012). Peak serum: implications of serum supply for cell therapy manufacturing. Regen Med 7, 7-13.

Coecke, S., Balls, M., Bowe, G., et al. (2005). Guidance on good cell culture practice. a report of the second ECVAM task force on good cell culture practice. Altern Lab Anim 33, 261-287.

Chong, S., Dando, S. A., and Morrison, R. A. (1997). Evaluation of Biocoat intestinal epithelium differentiation environment (3-day cultured Caco-2 cells) as an absorption screening model with improved productivity. Pharm Res 14, 1835-1837.

Cross, H. S. and Quaroni, A. (1991). Inhibition of sucrase-isomaltase expression by EGF in the human colon adenocarcinoma cells Caco-2. Am J Physiol 261, C1173-1183.

Delie, F. and Rubas, W. (1997). A human colonic cell line sharing similarities with enterocytes as a model to examine oral absorption: advantages and limitations of the Caco-2 model. Crit Rev Ther Drug Carrier Syst 14, 221-286.

Ferruzza, S., Scarino, M. L., Gambling, L., et al. (2003). Biphasic effect of iron on human intestinal Caco-2 cells: early effect on tight junction permeability with delayed onset of oxidative cytotoxic damage. Cell Mol Biol (Noisy-le-grand) 49, 89-99.

Ferruzza, S., Rossi, C., Scarino, M. L., and Sambuy,Y. (2012a). A protocol for in situ enzyme assays to assess the differentiation of human intestinal Caco-2 cells. Toxicol In Vitro 26, 1247-1251.

Ferruzza, S., Rossi, C., Scarino, M. L., and Sambuy,Y. (2012b). A protocol for differentiation of human intestinal Caco-2 cells in asymmetric serum-containing medium. Toxicol In Vitro 26, 1252- 1255 .
Fukuda, H., Arai, M., Soh, T., et al. (2006). Progesterone regulation of the expression and function of multidrug resistance type I in porcine granulosa cells. Reprod Toxicol 22, 62-68.

Gstraunthaler, G., Seppi, T., and Pfaller, W. (1999). Impact of culture conditions, culture media volumes, and glucose content on metabolic properties of renal epithelial cell cultures. Are renal cells in tissue culture hypoxic? Cell Physiol Biochem 9, 150-172.

Halleux, C. and Schneider, Y. J. (1991). Iron absorption by intestinal epithelial cells: 1 . Caco-2 cells cultivated in serumfree medium, on polyethyleneterephthalate microporous membranes, as an in vitro model. In Vitro Cell Dev Biol 27A, 293-302.

Hashimoto, K. and Shimizu, M. (1993). Epithelial properties of human intestinal Caco-2 cells cultured in a serum-free medium. Cytotechnology 13, 175-184.

Hidalgo, I. J., Raub, T. J., and Borchardt, R. T. (1989). Characterization of the human colon carcinoma cell line (Caco-2) as a model system for intestinal epithelial permeability. Gastroenterology 96, 736-749.

Hilgendorf, C., Ahlin, G., Seithel, A., et al. (2007). Expression of thirty-six drug transporter genes in human intestine, liver, kidney, and organotypic cell lines. Drug Metab Dispos 35, 1333-1340.

Hirsch-Ernst, K. I., Ziemann, C., Schmitz-Salue, C., et al. (1995). Modulation of P-glycoprotein and mdr1b mRNA expression by growth factors in primary rat hepatocyte culture. Biochem Biophys Res Commun 215, 179-185.

Hodin, R. A., Chamberlain, S. M., and Meng, S. (1995). Pattern of rat intestinal brush-border enzyme gene expression changes with epithelial growth state. Am J Physiol 269, C385-391.

Hunter, J., Hirst, B. H., and Simmons, N. L. (1993). Drug absorption limited by P-glycoprotein-mediated secretory drug transport in human intestinal epithelial Caco-2 cell layers. Pharm Res 10, 743-749.

Jumarie, C. and Malo, C. (1991). Caco-2 cells cultured in serum-free medium as a model for the study of enterocytic differentiation in vitro. J Cell Physiol 149, 24-33.

Jumarie, C. and Malo, C. (1994). Alkaline phosphatase and peptidase activities in Caco-2 cells: differential response to triiodothyronine. In Vitro Cell Dev Biol Anim 30A, 753-760.

Jumarie, C., Herring-Gillam, F. E., Beaulieu, J. F., and Malo, C. (1996). Triiodothyronine stimulates the expression of sucrase-isomaltase in Caco-2 cells cultured in serum-free medium. Exp Cell Res 222, 319-325.

Le Ferrec, E., Chesné, C., Artursson, P., et al. (2001). In Vitro Models of the Intestinal Barrier. ECVAM Workshop Report 46, Altern Lab Anim 29, 649-668

Lowry, O. H., Rosebrough, N. J., Farr, A. L., and Randall, R.J. (1951). Protein measurement with the Folin phenol reagent. $J$ Biol Chem 193, 265-275.

Madara, J. L. (1998). Regulation of the movement of solutes across tight junctions. Annu Rev Physiol 60, 143-159.

Matsumoto, H., Erickson, R. H., Gum, J. R., et al. (1990). Biosynthesis of alkaline phosphatase during differentiation of the 
human colon cancer cell line Caco-2. Gastroenterology 98, 1199-1207.

Miret, S., Abrahamse, L., and de Groene, E. M. (2004). Comparison of in vitro models for the prediction of compound absorption across the human intestinal mucosa. J Biomol Screen 9, 598-606.

Morel, E., Demignot, S., Chateau, D., et al. (2004). Lipid-dependent bidirectional traffic of apolipoprotein B in polarized enterocytes. Mol Biol Cell 15, 132-141.

Natoli, M., Leoni, B. D., D’Agnano, I., et al. (2011). Cell growing density affects the structural and functional properties of Caco-2 differentiated monolayer. J Cell Physiol 226, 15311543.

Natoli, M., Leoni, B. D., D’Agnano, I., et al. (2012). Good Caco-2 cell culture practices. Toxicol In Vitro 26, 1243-1246.

Nielsen, C. U., Amstrup, J., Nielsen, R., et al. (2003). Epidermal growth factor and insulin short-term increase hPEPT1-mediated glycylsarcosine uptake in Caco-2 cells. Acta Physiol Scand 178, 139-148.

Nielsen, C. U. and Brodin, B. (2003). Di/tri-peptide transporters as drug delivery targets: regulation of transport under physiological and patho-physiological conditions. Cur. Drug Targets 4, 373-388.

Pinto, M., Robine-Leon, S., Appay, M. D., et al. (1983). Enterocyte-like differentiation and polarization of the human colon carcinoma cell line Caco-2 in culture. Biol Cell 47, 323-330.

Ranaldi, G., Consalvo, R., Sambuy, Y., and Scarino, M.L. (2003). Permeability characteristics of parental and clonal human intestinal Caco-2 cell lines differentiated in serumsupplemented and serum-free media. Toxicol In Vitro 17, 761-767.

Rolleston, W. B. (1999). Bovine serum: reducing the variables through the use of donor herds. Dev Biol Stand 99, 79-86.

Sambuy, Y., De Angelis, I., Ranaldi, G., et al. (2005). The Caco-2 cell line as a model of the intestinal barrier: influence of cell and culture-related factors on Caco- 2 cell functional characteristics. Cell Biol Toxicol 21, 1-26.

Stephens, R. H., O’Neill, C. A., Warhurst, A., et al. (2001). Kinetic profiling of P-glycoprotein-mediated drug efflux in rat and human intestinal epithelia. J Pharmacol Exp Ther 296, 584-591.

Sun, D., Lennernas, H., Welage, L. S ., et al. (2002). Comparison of human duodenum and Caco-2 gene expression profiles for
12,000 gene sequences tags and correlation with permeability of 26 drugs. Pharm Res 19, 1400-1416.

Van Beers, E. H., Al, R. H., Rings, E. H., et al. (1995). Lactase and sucrase-isomaltase gene expression during Caco-2 cell differentiation. Biochem J 308 (Pt 3), 769-775.

van der Valk, J., Mellor, D., Brands, R., et al. (2004). The humane collection of fetal bovine serum and possibilities for serum-free cell and tissue culture. Toxicol In Vitro 18, 1-12.

van der Valk, J., Brunner, D., De Smet, K., et al. (2010). Optimization of chemically defined cell culture media--replacing fetal bovine serum in mammalian in vitro methods. Toxicol In Vitro 24, 1053-1063.

Van Itallie, C. M., Holmes, J., Bridges, A., et al. (2008). The density of small tight junction pores varies among cell types and is increased by expression of claudin-2. J Cell Sci 121, 298-305.

Walter, E. and Kissel, T. (1995). Heterogeneity in the human intestinal cell line Caco- 2 leads to differences in transepithelial transport. Eur J Pharm Sci 3, 215-230.

Yamashita, S., Konishi, K., Yamazaki, Y., et al. (2002). New and better protocols for a short-term Caco-2 cell culture system. $J$ Pharm Sci 91, 669-679.

Zucco, F., Batto, A. F., Bises, G., et al. (2005). An inter-laboratory study to evaluate the effects of medium composition on the differentiation and barrier function of Caco-2 cell lines. Altern Lab Anim 33, 603-618.

\section{Acknowledgements}

Work supported by EU FP6 Project "LIINTOP" contract number STREP-037499. For cephalexin determination we wish to thank Mirjam Spansier, Janine Zwerink, Aline Bloemendal, and Martijn Rooseboom while working at Schering-Plough, Oss, The Netherlands as LIINTOP project partners.

\section{Correspondence to}

Maria Laura Scarino, $\mathrm{PhD}$

National Research Institute on Food and Nutrition (INRAN)

Via Ardeatina 546

00178 Rome

Italy

e-mail: scarino@inran.it 